THRESHOLD BUBBLE CHAMBER FOR MEASUREMENT OF KNOCK-ON DT NEUTRON TAILS FROM MAGNETIC AND INERTIAL CONFINEMENT EXPERIMENTS

by R.K. FISHER, V.S. ZAVERYAEV, AND S.V. TRUSILLO

JULY 1996 


\section{DISCLAIMER}

This report was prepared as an account of work sponsored by an agency of the United States Government. Neither the United States Government nor any agency thereof, nor any of their employees, makes any warranty, express or implied, or assumes any legal liability or responsibility for the accuracy, completeness, or usefulness of any information, apparatus, produce, or process disclosed, or represents that its use would not infringe privately owned rights. Reference herein to any specific commercial product, process, or service by trade name, trademark, manufacturer, or otherwise, does not necessarily constitute or imply its endorsement, recommendation, or favoring by the United States Government or any agency thereof. The views and opinions of authors expressed herein do not necessarily state or reflect those of the United States Government or any agency thereof. 


\section{DISCLAIMER}

Portions of this document may be illegible in electronic image products. Images are produced from the best available original document. 


\title{
THRESHOLD BUBBLE CHAMBER FOR MEASUREMENT OF KNOCK-ON DT NEUTRON TAILS FROM MAGNETIC AND INERTIAL CONFINEMENT EXPERIMENTS
}

\author{
by \\ R.K. FISHER, V.S. ZAVERYAEV, ${ }^{\star}$ AND S.V. TRUSILLO†
}

This is a preprint of an invited paper to be presented at the 11th Topical High Temperature Plasma Diagnostic Conference, May 12-16, 1996, Monterey, California and to be published in Review of Scientific Instruments

\footnotetext{
"Russian Research Centre, "Kurchatov Institute," Nuclear Fusion Institute, Moscow, Russian Federation $t_{\text {Russian Federal Nuclear Centre, "Experimental Physics }}$ Institute," Arzamas, Russian Federation
}

Work supported by the U.S. Department of Energy under Grant No. DE-FG03-92ER54150

GA PROJECT 3937 JULY 1996 


\title{
Threshold Bubble Chamber for Measurement of Knock-on DT Neutron Tails from Magnetic and Inertial Confinement Experiments
}

\author{
R.K. Fisher \\ General Atomics, P.O. Box 85608, San Diego, California
}

\section{V.S. Zaveryaev}

Russian Research Centre, "Kurchatov Institute," Nuclear Fusion Institute, Moscow, Russian Federation

\section{S.V. Trusillo}

Russian Federal Nuclear Centre, "Experimental Physics Institute," Arzamas, Russian Federation

We propose a new "threshold" bubble chamber detector for measurement of knock-on neutron tails. These energetic neutrons result from fusion reactions involving energetic fuel ions created by alpha knock-on collisions in tokamak and other magnetic confinement experiments, and by both alpha and neutron knock-on collisions in inertial confinement fusion (ICF) experiments. The energy spectrum of these neutrons will yield information on the alpha population and energy distribution in tokamaks, and on alpha target physics and $\rho R$ measurements in ICF experiments. The bubble chamber should only detect neutrons with energies above a selectable threshold energy controlled by the bubble chamber pressure. The bubble chamber threshold mechanism, detection efficiency, and proposed applications to the International Thermonuclear Experimental Reactor (ITER) and National Ignition Facility (NIF) experiments will be discussed. 


\section{INTRODUCTION}

Fusion ignition requires that the $3.5 \mathrm{MeV} \alpha$ particles from deuteriumtritium reactions deposit a large fraction of their energy in the reacting plasma before they are lost. In magnetic confinement experiments such as the proposed International Thermonuclear Experimental Reactor (ITER), the DT $\alpha$ 's must be confined for a time scale longer than their classical slowing-down time $(\sim 1 \mathrm{sec})$. In inertial confinement (ICF) experiments such as the planned National Ignition Facility (NIF), the plasma density must be large enough to slow down or stop the $\alpha$ 's before they can escape from the target plasma.

Because of the importance of $\alpha$-particles in fusion research, a number of diagnostic techniques are under development. 1 One of the few $\alpha-$ diagnostic techniques which is applicable to both tokamak and ICF experiments utilizes the energetic DT neutron tail resulting from $\alpha-$ particle collisions with the plasma fuel ions.

\section{PRODUCTION OF DT NEUTRON TAILS}

The elastic scattering collisions that transfer the $\alpha$ energy to the DT plasma and allow fusion ignition will also create a high energy tail on the deuterium and tritium ion energy distributions. ${ }^{2-3}$ An $\alpha$-particle can create a $3.4 \mathrm{MeV}$ tritium ion or a $3.1 \mathrm{MeV}$ deuterium ion in a single collision. Figure l(a) shows the calculated tritium ion energy distribution in a tokamak under plasma conditions similar to those expected in the ITER tokamak, $\mathrm{n}_{\mathrm{e}}=2 \mathrm{n}_{\mathrm{d}}=2 \mathrm{n}_{\mathrm{t}}=8 \times 10^{13} \mathrm{~cm}^{-3}$ and $\mathrm{T}_{\mathrm{i}}=\mathrm{T}_{\mathrm{e}}=17 \mathrm{keV}$. The $\alpha$-energy distribution is assumed to be the classical slowing-down 

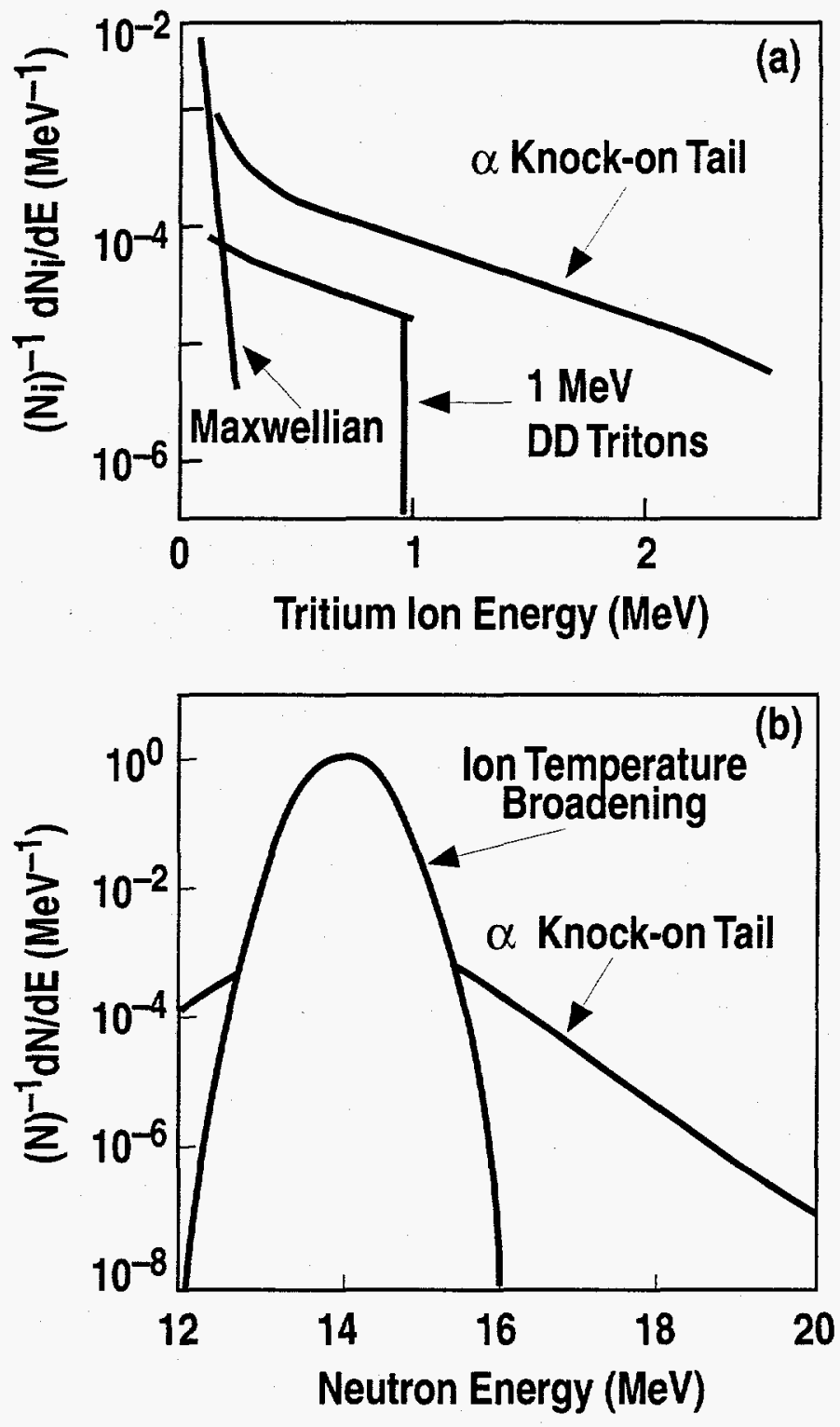

Fig. 1. Calculated energy spectra of (a) tritium plasma ions and (b) DT neutrons showing knock-on tails resulting from alpha particle collisions in ITER plasma at $n_{e}=2 n_{d}=2 n_{t}=8 \times 10^{13} \mathrm{~cm}^{-3}$ and $T_{i}=T_{e}=17 \mathrm{keV}$.

distribution characteristic of well-confined $\alpha$ 's. ${ }^{4}$ Due to the small impact parameter during knock-on collisions, the nuclear plus interference forces dominate over the Coulomb force. 
The alpha-induced knock-on tail becomes larger than the Maxwellian thermal ion population at tritium ion energies above $250 \mathrm{keV}$. Also shown in Fig. 1(a) is the classical slowing-down distribution for the $1 \mathrm{MeV}$ tritons from $\mathrm{D}-\mathrm{D}$ fusion reactions. Fortunately, the alpha-induced knock-on tail is several times larger than the D-D triton population under ITER-like conditions and, hence, measurement of the ion tail can be used to learn about the $\alpha$ 's.

Some of the resulting energetic fuel ions will undergo DT fusion reactions with the background plasma, producing neutrons whose energy is significantly above $14 \mathrm{MeV}$ due to the excess kinetic energy of the reacting ions in the center-of-mass frame. Figure l(b) from Ref. 3 shows the expected DT neutron energy distribution resulting from the tritium ion tail of Fig. 1(a). Above $\sim 15.5 \mathrm{MeV}$, the DT neutron energy spectrum is determined by the $\alpha$ knock-on collision effects. The magnitude of the neutron tail is linearly proportional to the $\alpha$ population at the spatial location where the neutrons are generated. Measurements of the neutron energy spectrum can be unfolded to obtain information on an $\alpha$ energy spectrum. Note that the knock-on neutron tail is very small, with only $\sim 2 \times 10^{-4}$ of the total DT neutrons above $15.5 \mathrm{MeV}$ for a tokamak under ITER-like conditions.

In an ICF experiment, an energetic neutron tail will be created by the same alpha knock-on collisions with fuel ions, but a comparable size tail can also be created by the DT neutrons through knock-on collisions with the fuel ions. A $14 \mathrm{MeV}$ neutron can create a deuterium ion up to $\sim 12.5 \mathrm{MeV}$ in a single collision, which can subsequently produce DT neutrons with energies up to $\sim 30 \mathrm{MeV} .5$ Hence, the knock-on neutron tail, called "tertiary" neutrons in ICF experiments, will reflect the $\alpha$ - 
induced collisions at neutron energies below $\sim 20.6 \mathrm{MeV}$, the maximum neutron energy from a $3.5 \mathrm{MeV}$ alpha-induced collision, and will reflect the neutron-induced collisions at neutron energies below $30.1 \mathrm{MeV}$. Since the $\alpha$-fuel ion and $n$-fuel ion elastic scattering cross sections are comparable, both processes will contribute below $20.6 \mathrm{MeV}$. The production of tertiary neutrons in ICF experiments can be used to measure the fuel areal density $\rho R$, an important parameter in ICF experiments, since it reflects whether the fuel density is compressed sufficiently to slow down the $\alpha$ 's and allow fusion ignition. The calculated neutron tail is again very small. The fraction of the total DT neutron flux which is above $16 \mathrm{MeV}$ is $\sim 6 \times 10^{-4}\langle\rho R\rangle^{2}$ where $\langle\rho R\rangle$ is in $\mathrm{gm} / \mathrm{cm}^{2}{ }^{4}$ The $\langle\rho \mathrm{R}\rangle^{2}$ scaling is due to the fact that the probability that a neutron or alpha elastically scatters to produce an energetic fuel ion and the probability that subsequently the recoil ion will react with the colder fuel are both proportional to $\langle\rho R\rangle$ for small $\langle\rho R\rangle$. For the $\langle\rho R\rangle$ $\sim 50 \mathrm{mg} / \mathrm{cm}^{2}$ achieved in existing ICF experiments, the neutron tail is about $10^{-6}$ of the neutron flux. On NIF, where $\langle\rho R\rangle$ may approach $100 \mathrm{gm} / \mathrm{cm}^{3}$, the tail will be much larger, although the $\langle\rho R\rangle^{2}$ formulation above will no longer be valid.

\section{THRESHOLD BUBBLE CHAMBER}

Previous studies examined a number of neutron spectroscopy techniques for knock-on tail measurements, 2,6 with magnetic proton recoil spectrometers, ${ }^{7}$ time-of-flight spectrometers, $, 8,9$ and threshold neutron activation detectors ${ }^{2}$ as the most attractive approaches. 
This paper proposes a new approach, the threshold bubble chamber, which appears to be an almost ideal candidate detector for measurement of the knock-on neutron tails from both tokamak and ICF target plasmas. It should be nearly totally insensitive to the much larger flux of $14 \mathrm{MeV}$ neutrons from the plasma and gamma-rays created by $(\mathrm{n}, \gamma)$ reactions in the surrounding structures; and yet have a high detection efficiency for neutrons above a threshold energy which is controlled by the chosen bubble chamber pressure.

The bubble chamber uses carbon tetrachloride, $\mathrm{CCl}_{4}$, as its working liquid and operates at room temperature and at pressures of $\sim 0.5 \mathrm{~atm}$, and hence avoids the complications of cooling or high pressure operation. There is no requirement for an external magnetic field on the bubble chamber since there are no charged particle tracks to measure. The information on the neutron energy comes entirely from the neutron threshold energy condition.

The neutron energy threshold results in the incident neutrons either creating zero or one bubble. Bubble production requires that sufficient heat (energy $\sim 1000$ s of $\mathrm{eV}$ ) be deposited in a very short distance $\left(\sim 10^{-6} \mathrm{~cm}\right) .{ }^{10}$ The bubble formation only involves a few thousand atoms. Only the recoil chlorine ions resulting from near head-on collisions with neutrons produce bubbles as described below.

The incident neutrons collide with the atoms in the carbon tetrachloride bubble chamber liquid, creating recoil chlorine and carbon ions of energy

$$
E_{A}=E_{n}\left(4 A \cos ^{2} \theta_{A}\right) /(1+A)^{2}
$$

where $A$ is the mass of the recoil ion and $\theta_{\mathrm{A}}$ is the angle of emission of the recoil ion with respect to the incident neutron direction. Figure 2 


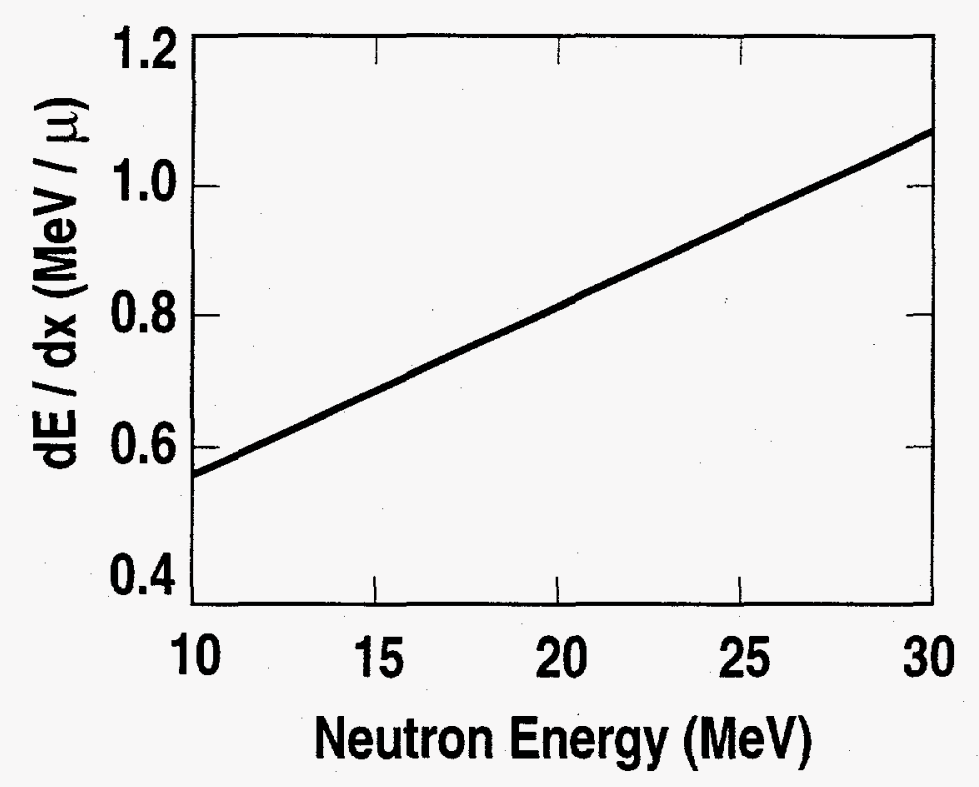

Fig. 2. Specific energy loss for the maximum energy recoil ${ }^{35} \mathrm{Cl}$ ion versus the incident neutron energy.

shows the specific energy loss $\mathrm{dE} / \mathrm{dx}$ in $\mathrm{MeV}$ per micron for the maximum energy recoil chlorine ion that can result from a head-on collision $\left(\theta_{\mathrm{A}}=0^{\circ}\right)$ as a function of the incident neutron energy. Note that $\mathrm{E}_{\mathrm{Cl}}^{\max } \cong 0.11 \mathrm{E}_{\mathrm{n}}$ based on Eq. (1). The recoil carbon ions that also result from neutron collisions, although more energetic than chlorine due to their lower $\mathrm{A}$, do not produce bubbles because their $\mathrm{dE} / \mathrm{dx}$ is too small. Similarly, the recoil electrons or delta-rays normally associated with bubble and track production in more conventional bubble chambers do not have sufficient energy to produce bubbles. Hence, only the recoil chlorine ions can create bubbles. Since $\mathrm{dE} / \mathrm{dx}$ is an increasing function of the recoil chlorine ion energy in this energy range, only neutrons above the threshold energy can produce bubbles.

Using the maximum available heat deposition as a function of neutron energy shown in Fig. 2, one can calculate from the bubble formation 
physics, the bubble chamber pressure required so that bubbles can only result from neutrons at or above that energy. This threshold pressure is shown as a function of the desired neutron threshold energy in Fig. 3, for $\mathrm{CCl}_{4}$ at room temperature. Fortunately, the threshold pressure falls in a workable range near 0.5 atmosphere. At a given pressure, the neutron energy threshold is very short, estimated to be on the order of $10 \mathrm{keV}$, based on the small variations in the specific energy loss for the record chlorine ions and in the energy required to form a bubble.

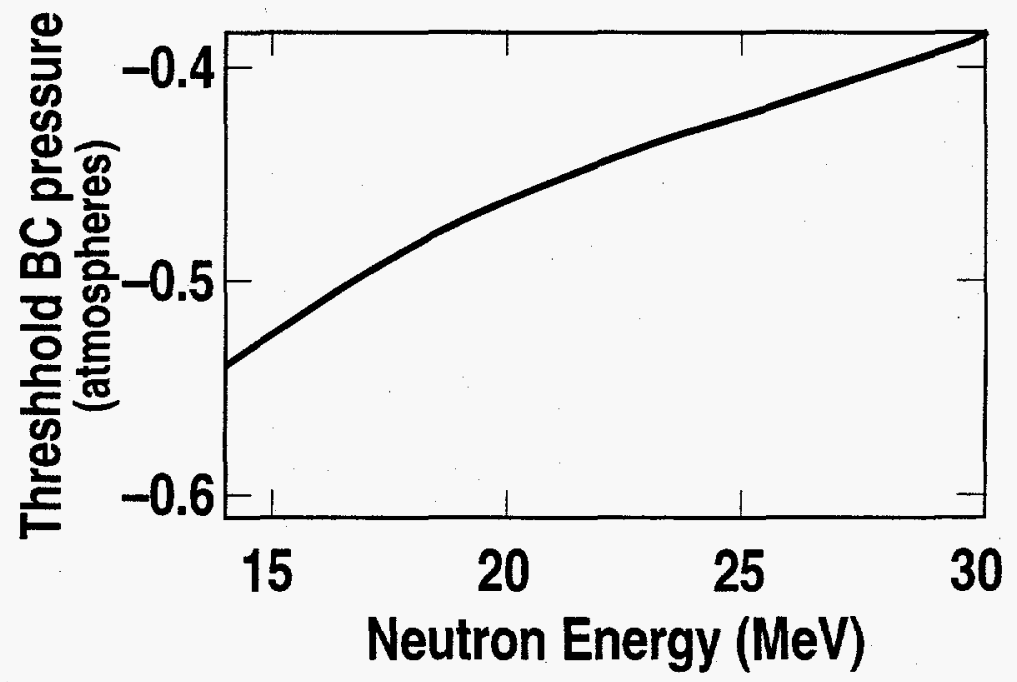

Fig. 3. Calculated threshold pressure for $\mathrm{CCl}_{4}$ bubble chamber as a function of desired neutron threshold energy.

The threshold bubble chamber is also nearly insensitive to the large background flux of gamma-rays resulting from neutron capture reactions in structures surrounding the tokamak or ICF plasmas. Gamma-rays of energies up to $14 \mathrm{MeV}$ will produce electrons via pair-production and or Compton scattering. But the resultant electrons have a $\mathrm{dE} / \mathrm{dx}$ which is too small to create bubbles. In a test of a similar bubble chamber ${ }^{11}$ used to detect $2.5 \mathrm{MeV}$ neutrons from D-D plasmas, the measured gamma sensitivity was $\sim 10^{-12}$ of the neutron sensitivity. 
The neutron detection efficiency $\varepsilon_{\mathrm{BC}}$ can be fairly large, and is equal to $\varepsilon_{B C}=P_{R} P_{B}$ where $P_{R}$ is the probability of creating a sufficiently energetic recoil chlorine ion and $\mathrm{P}_{\mathrm{B}}$ is the probability that the chlorine ion will subsequently produce a bubble. Since $P_{B}=100 \%, \varepsilon_{B C}$ is just the probability of a neutron creating a recoil chlorine ion of sufficient energy $\mathrm{E}_{\mathrm{A}}^{\text {th }}$ to produce a bubble

$$
\varepsilon_{\mathrm{BC}} \simeq \mathrm{n}_{\mathrm{CL}} \Delta \mathrm{x} \int_{0}^{\theta_{\mathrm{A}}^{\max }}(\mathrm{d} \sigma / \mathrm{d} \Omega) \mathrm{d} \Omega,
$$

where $\mathrm{n}_{\mathrm{Cl}}$ is the density of chlorine atoms, $\Delta \mathrm{x}$ is the overall thickness of the bubble chamber along the neutron path, $d \sigma / d \Omega$ is the differential cross section for neutrons elastically scattering from chlorine near $\theta_{\mathrm{A}} \simeq$ $0^{\circ}\left(\theta_{\mathrm{n}} \simeq 180^{\circ}\right)$, and $\theta_{\mathrm{A}}^{\max }$ is the maximum scattering angle for a collision producing a chlorine ion of energy $\mathrm{E}_{\mathrm{A}}^{\text {th }}$. Equation (1) gives $\cos \theta_{\mathrm{A}}^{\max } \simeq$ $1 / 2\left(\Delta E / E_{n}\right)$ where $\Delta E \equiv E_{n}-E_{n}^{\text {th }} \ll E_{n}$ and $E_{n}^{\text {th }}$ is the neutron threshold energy, the minimum neutron energy required to produce a chlorine ion of energy $E_{A}^{\text {th }}$. Since it does little good for the bubble chamber thickness to be larger than the mean free path of the incident neutrons, we take $\Delta \mathrm{x}=\left(\mathrm{n}_{\mathrm{Cl}} \sigma_{\mathrm{tot}}\right)^{-1}$ where $\sigma_{\mathrm{tot}}$ is the total neutron scattering cross section. Hence,

$$
\varepsilon_{\mathrm{BC}} \simeq\left(\sigma_{\mathrm{tot}}\right)^{-1}(\mathrm{~d} \sigma / \mathrm{d} \Omega) \pi\left(\Delta \mathrm{E} / \mathrm{E}_{\mathrm{n}}\right)
$$

yielding

$$
\varepsilon_{\mathrm{BC}} \simeq 0.06\left(\Delta \mathrm{E} / \mathrm{E}_{\mathrm{n}}\right)
$$

for neutron energies near $16 \mathrm{MeV}$.

For $E_{n}=16 \mathrm{MeV}$ and $E_{n}^{\text {th }}=15.5 \mathrm{MeV}$, the probability of creating a recoil chlorine ion above required threshold energy is

$$
\varepsilon_{\mathrm{BC}}=2 \times 10^{-3} \text {. }
$$




\section{APPLICATION TO TOKAMAKS}

By installing an array of threshold bubble chamber detectors behind a neutron collimator, similar to the geometry sketched in Fig. 4 for ITER, it should be possible to measure the $\alpha$ density profile. Each channel of the array will measure the knock-on neutron tail integrated over the detector sight line. If the neutron emission profile is strongly peaked, the knockon tail for a given channel will primarily reflect the local $\alpha$ density at the position of maximum neutron emission along that sight line, i.e., at the point of tangency to the plasma flux surface intersecting that sight line. Hence, the geometry shown in Fig. 4 would primarily measure the vertical $\alpha$ density profile. By measuring the knock-on neutron tail as a function of energy, it should also be possible to gain information about the $\alpha$ energy spectrum.

The number of bubbles produced per unit frontal area of the bubble chamber is given by

$$
\mathrm{dNB} / \mathrm{dA}=\mathrm{f}_{\text {tail }} \dot{\Phi}_{\mathrm{DT}} \varepsilon_{\mathrm{BC}} \Delta \mathrm{t}
$$

where $f_{\text {tail }} \sim 2 \times 10^{-4}$ is the fraction of the total DT neutrons above 15.5 MeV on ITER; $\dot{\Phi}_{\mathrm{DT}}$ is the total DT neutron flux at the detector, $\varepsilon_{\mathrm{BC}}$ is the detection efficiency and $\Delta t$ is the total data collection time.

Because the bubbles grow and disappear on a time scale of less than $10^{-3} \mathrm{sec}$, the bubble chamber pressure should be cycled at several $\mathrm{kHz}$. Since the neutron signal from a tokamak will be integrated over many pressure cycles, the average neutron detection efficiency will be reduced by a "duty cycle" fraction $f_{D C}$, the fraction of the overall cycle time that the bubble chamber pressure is appropriate $\left(E_{n}^{\text {th }}<E_{n}\right.$ of interest). Depending on whether sine wave or square wave pressure cycling is 


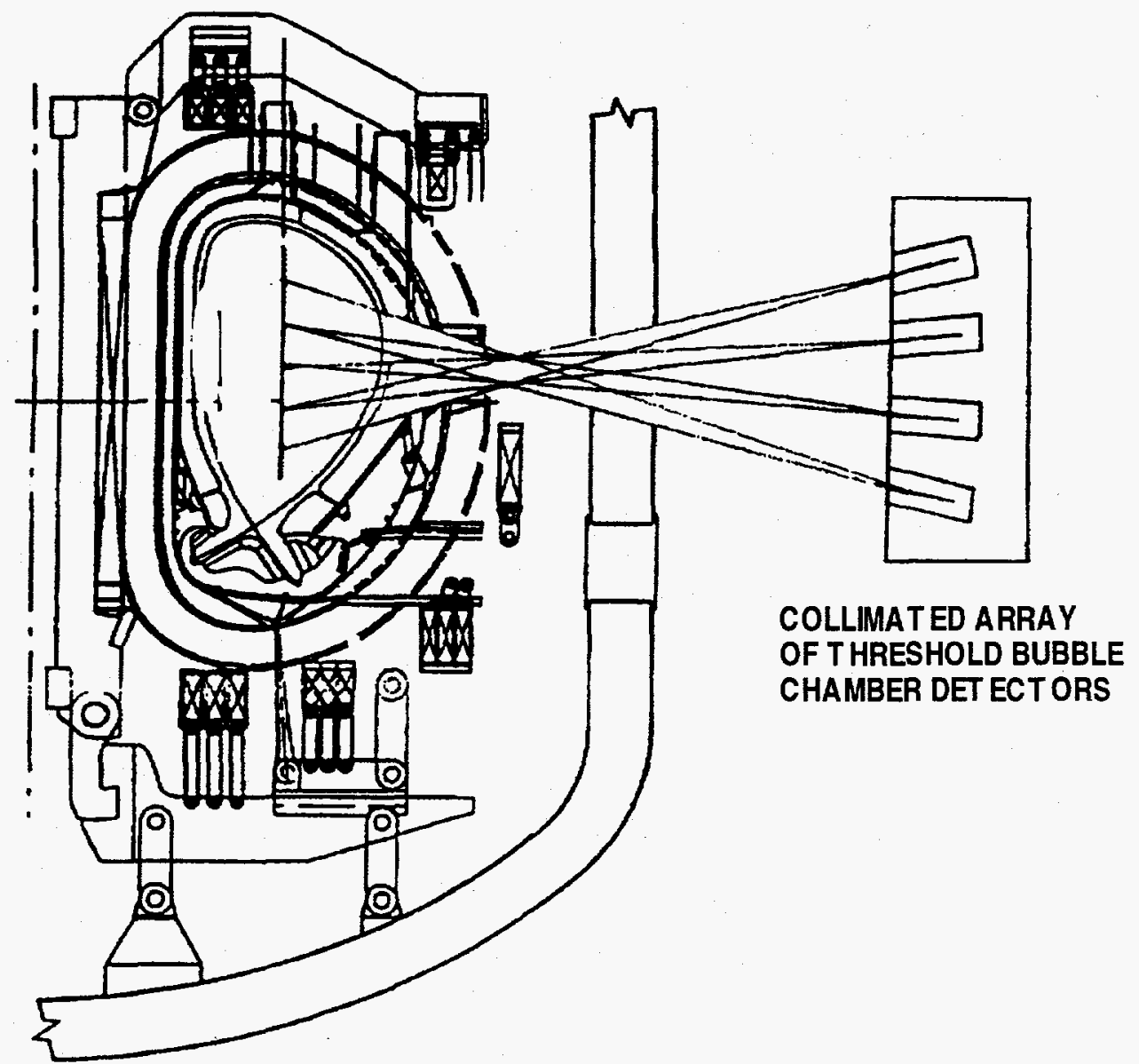

Fig. 4. Schematic showing possible array of threshold bubble chamber detectors behind a neutron collimator for measurement of the spatial profile of $n_{\alpha}(r)$ and $d n_{\alpha} / d E$ on ITER.

used, $\mathrm{f}_{\mathrm{DC}} \sim 0.03$ to 0.5 , so that $\left\langle\varepsilon_{\mathrm{BC}}\right\rangle=\mathrm{f}_{\mathrm{DC}} \varepsilon_{\mathrm{BC}} \simeq 10^{-4}$ to $10^{-3}$ for a tokamak experiment. The physics limit on the time resolution for the knock-on approach to alpha diagnostics in tokamaks is equal to the tritium ion slowing-down time of $\sim 3 \mathrm{sec}$ under ITER plasma conditions. Even if all the alphas were rapidly lost due to an instability, the tritium ion tail would only decay away on a 3-sec time scale unless affected by the same instability mechanism that affected the alphas. 
Usind $\Delta \mathrm{t}=3 \mathrm{sec},\left\langle\varepsilon_{\mathrm{BC}}\right\rangle=10^{-3}$ and $\mathrm{f}_{\text {tail }}=2 \times 10^{-4}$ above $15.5 \mathrm{MeV}$ in Eq. (7), a knock-on signal of one bubble per $\mathrm{cm}^{2}$ of bubble chamber frontal area requires a total DT incident neutron flux of only $2 \times$ $10^{6} \mathrm{n} / \mathrm{cm}^{2}$-sec. This is well below the neutron flux inside the planned neutron cameras on ITER, which is expected to be in the range of several times $10^{8}$ to $10^{9} \mathrm{n} / \mathrm{cm}^{2}$-sec. Hence, an array of threshold bubble chamber detectors could be installed as part of the detector arrays in the ITER neutron cameras. It would provide critical information on $n_{\alpha}(r)$ and $\mathrm{dn}_{\alpha} / \mathrm{dE}$.

Since the knock-on neutron signal is proportional to $\mathrm{n}_{\alpha} \tau_{\mathrm{sd}} \dot{\Phi}_{\mathrm{DT}}$, and hence falls very rapidly in the off-axis spatial channels or in lower power ITER discharges, it would be best for the threshold $\mathrm{BC}$ detectors to be in a separate neutron collimator array which could be part of the neutron camera system shield, but with a larger pinhole aperture. To maximize the knock-on signals, the knock-on detector array should have the largest possible pinhole aperture consistent with the desired spatial resolution. It would not require a substantial neutron collimator/shield because any scattered neutron would be below the neutron threshold energy. Hence, even a totally separate shield/collimator would be much less costly and space consuming than the conventional neutron camera arrays.

The threshold bubble chamber could be tested on TFTR or JET since these experiments will produce a similar size tail fraction and sufficient DT neutron flux. 


\section{APPLICATION TO ICF EXPERIMENTS}

The knock-on neutron tail on NIF should easily be large enough to produce measurable signals using threshold bubble chambers. In an ICF experiment, the neutrons are all created in a time short compared to the bubble growth time, so the bubble chamber data will be time integrated for each shot. Total neutron fluence per shot $\Phi_{\mathrm{DT}}=\dot{\Phi}_{\mathrm{DT}} \Delta \mathrm{t}$ becomes the parameter of interest and Eq. (4) becomes $\mathrm{dN}_{\mathrm{b}} / \mathrm{dA}=\mathrm{f}_{\text {tail }} \Phi_{\mathrm{DT}} \varepsilon_{\mathrm{BC}}$. Using $\varepsilon_{\mathrm{BC}}=10^{-3}$, a knock-on tail neutron fluence $\Phi_{\mathrm{k}-0}=\mathrm{f}_{\text {tail }} \Phi_{\mathrm{DT}}$ of $10^{3} \mathrm{n} / \mathrm{cm}^{2}$ will produce one bubble/ $/ \mathrm{cm}^{2}$ of bubble chamber frontal area. NIF is designed to produce $10^{18}$ total neutrons per shot, so that the DT neutron fluence $300 \mathrm{~cm}$ from the target will be $\Phi_{\mathrm{DT}}=9 \times 10^{11} \mathrm{n} / \mathrm{cm}^{2}$. Hence, an $\mathrm{f}_{\text {tail }}$ as small as $10^{-9}$ would be measurable. On the other hand, NOVA generates $\leqslant 10^{9}$ DT neutrons/shot and $\leqslant 10^{4}$ knock-on tail neutrons/shot which is insufficient to produce a useful knock-on signal. The threshold bubble chamber detector could be tested on NOVA by viewing the $14 \mathrm{MeV}$ neutrons from $1 \mathrm{MeV} \mathrm{D}-\mathrm{D}$ tritons interacting with the cold D fuel to measure $\langle\rho \mathrm{R}\rangle$. Because NIF will generate knock-on tails from both alpha-induced and neutron-induced elastic scattering collisions, information on $\langle\rho R\rangle$. and on alpha interaction physics should be possible.

As in tokamak experiments, information on the energy spectrum of the knock-on neutron tail can be obtained with a number of threshold bubble chambers, each one tuned to a different neutron energy threshold. 


\section{SUMMARY}

The threshold bubble chamber appears to be a nearly ideal neutron spectroscopy detector for measurement of the knock-on neutron tail from both tokamaks and ICF experiments. It should be almost totally insensitive to the large background of $14 \mathrm{MeV}$ neutrons and associated gamma-rays from DT plasmas, while having a high detection efficiency $\varepsilon_{\mathrm{BC}} \simeq 10^{-3}$ for the knock-on tail neutrons above $\sim 16 \mathrm{MeV}$. Easily measurable signals should yield information on $n_{\alpha}(r)$ and $d_{\alpha} / d E$ on ITER and on $\langle\rho R\rangle$ and alpha slowing-down on NIF.

\section{ACKNOWLEDGMENTS}

This work was supported by the U.S. Department of Energy under Grant No. DE-FG03-92ER54150.

\section{DISCLAIMER}

This report was prepared as an account of work sponsored by an agency of the United States Government. Neither the United States Government nor any agency thereof, nor any of their employees, makes any warranty, express or implied, or assumes any legal liability or responsibility for the accuracy, completeness, or usefulness of any information, apparatus, product, or process disclosed, or represents that its use would not infringe privately owned rights. Reference herein to any specific commercial product, process, or service by trade name, trademark, manufacturer, or otherwise does not necessarily constitute or imply its endorsement, recommendation, or favoring by the United States Government or any agency thereof. The views and opinions of authors expressed herein do not necessarily state or reflect those of the United States Government or any agency thereof. 
IB. Stratton, et al., this conference; F. Hoekzema, et al., this conference; R. K. Fisher, et al., this conference; R. J. Umstaatd, et al., this conference; J. S. Machuzak, et al., this conference, T. C. Sangster, et al., this conference; M. Moran, et al., this conference; R. Petrasso, et al., this conference; Proc. 11th Topical Conference on High-Temperature Plasma Diagnostics, May 12-16, 1996, Monterey, California.

${ }^{2}$ R. K. Fisher, et al., Nucl. Fusion 34, 1291 (1994).

${ }^{3}$ G. Gorini, et al., Rev. Sci. Instrum. 66, 936 (1995).

${ }^{4}$ H. P. Furth, et al., Nucl. Fusion 30, 1799 (1990).

${ }^{5}$ H. Azechi, M. D. Cable and R. O. Stapf, Lasers and Particle Beams 9, 119 (1991).

6R. K. Fisher, et al., "Diagnostics for Experimental Thermonuclear Reactors," P. E. Stott, G. Gorini, and E. Sindoni (eds.), Plenum Press, New York (ISBN 0-306-45297-3) (1996).

7J. Kallne and G. Gorini, Fusion Technology 25, 341 (1994).

8T. Elevant, Rev. Sci. Instrum. 66, 881 (1995).

${ }^{9}$ M. Moran, et al., this conference; R. E. Chrien, et al., this conference; T. J. Murphy, this conference.

10Y. A. Alexandrov, et al., "Bubble Chambers," Indiana University Press, Bloomington, Indiana (1967).

11S. V. Trusillo, et al., JETP Lett. 33, 148 (1981). 